\title{
Research on the Method of Spacecraft Orbit Determination Based the Technology of Dynamic Model Compensation
}

\author{
Pan Xiaogang, Wang Jiongqi and Zhou Haiyin \\ National University of Defence Technology, \\ China
}

\section{Introduction}

For the limit of dynamic model knowledge, model error will exist objectively in satellite dynamic model, especially for non-cooperation satellite, whose quality and shapes are unknown. While the precision of orbit determination results depends on the satellite dynamic model error, because dynamic model error will transmit to measurement data, and then mixed with measurement data error itself and result in the produce of the new system error data named Mixed Error(ME) in measurement model, which cannot be accurately described with parameters. The ME can be popularly dealt with by three methods: 1 . to regard ME as stochastic error. By analyzing the characteristic of error, it is chosen the corresponding estimator[1]. 2. Geometrical method for orbit determination. The dynamic model will not attend the process of orbit determination, so the ME doesn't exist [2]. 3. Reduced-dynamic orbit determination method. Process noise is added to dynamic model, which can absorb the dynamic model error[3.4]. Many scholars had researched the above orbit determination methods, and gained great success under given circumstance. For the first method, it is very difficult to determine the distribution of error data, so the precision of orbit determination will be influenced badly. Satellite dynamic model was avoided with the second method, the precision will not be affected by dynamic model, but it cannot forecast the satellite orbit and cannot analyze the orbit characteristic for geometrical method. For the third method, it was the most effective method to restrain the dynamic model error, but it needs precise measurement data (GPS) to restrict the model. It can be summarized from the above analysis that a new dynamic model error compensation method must be established to deal with the common orbit determination question. Semi-parametric model of orbit determination was established in this paper. Parametric function was used to describe the exact dynamic model, and non parametric function was founded to describe the ME. Because of the rationality of semi-parametric model, it gained great development since it was produced by Engle in 1980s. Many solve method of semi-parametric was researched such as regularization methd, B-spine compensation method and two stage method [5]. In traditional two stage method, nonlinear model regression was usually used to estimate the non parametric parts in semi-parametric model, but it can be distorted by the gross error, so the data depth theory was applied to semi-parametric model called Stahel -Donoho Kernel Estimator [6]. 
The precision of orbit determination depends on the precision of measurement data and the precision of dynamic model. In modern times, the degree of measurement can be limited in millimeter degree, so the key method to improve the precision of orbit determination is to increase the exactness of dynamic model, or to compensate the model error. In this chapter, some mathematic method are proposed to compensate the uncertainty model error, all those method is focus on the mathematics models, at last, a new orbit determination method based on model error compensation is put forward to deal with directly the dynamic force.

\section{Three different method of orbit determination based on dynamic model}

The dynamic model can describe the orbit character of satellite, and the position and velocity at time $t$ can be got by integral of dynamic model with condition of initial parameters. Based on the different method of state transfer and dynamic model the method of orbit determination is different too.

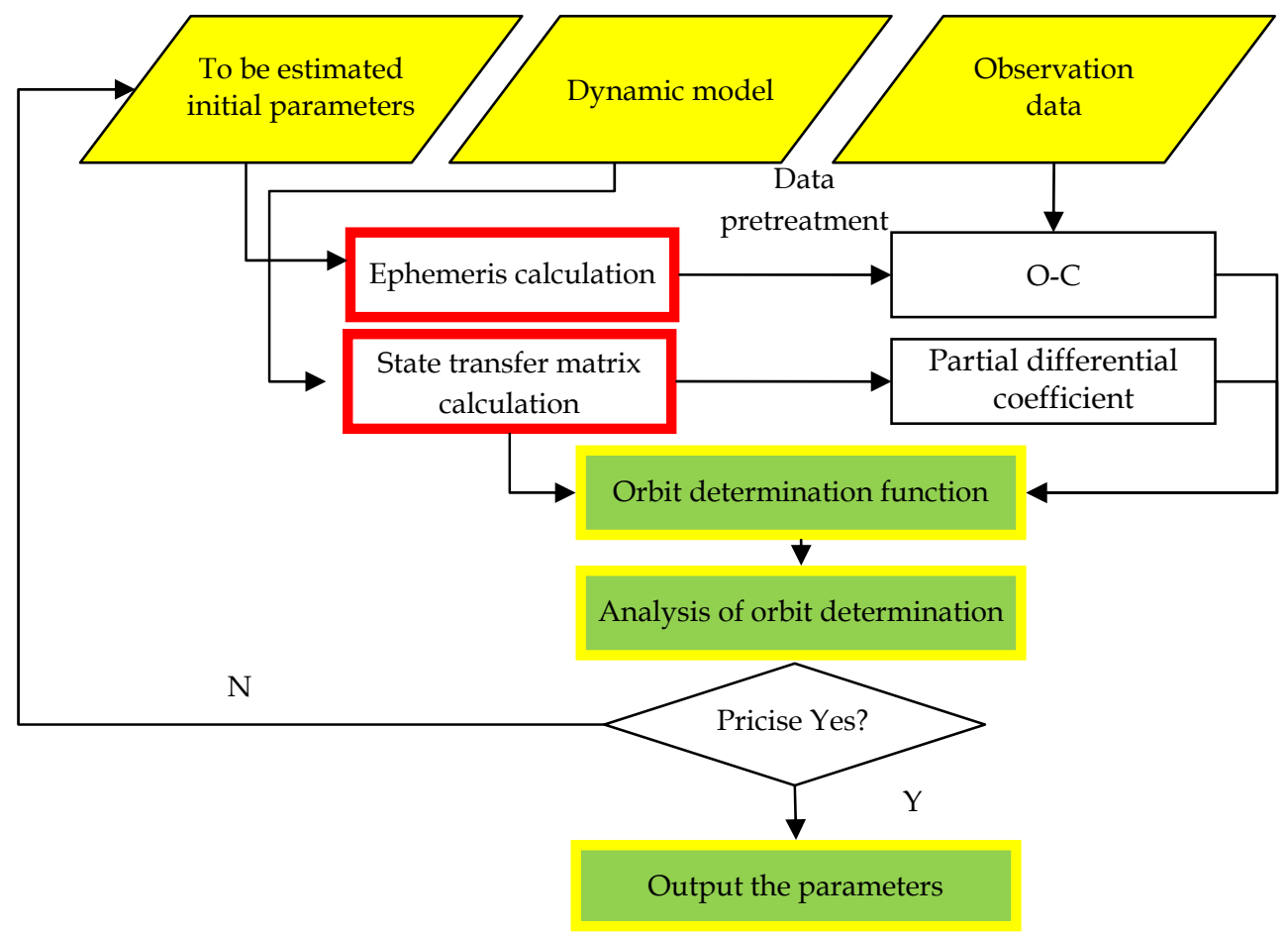

Fig. 1. Flow chart of orbit determination based on dynamic model.

\subsection{Theory of orbit determination based on transcendental information}

Based on the function of satellite, the dynamic model of satellite can be noted as

$$
\left\{\begin{array}{l}
\dot{X}(t)=f(X(t), t) \\
X\left(t_{0}\right)=X_{0}
\end{array}\right.
$$


Where, $X=\left(r, \dot{r}, p_{c}, p_{l}\right)^{\mathrm{T}}$ is to be improved state vector and model parameters. $r, \dot{r}$ is the position and velocity vector of satellite or the ephemeris of satellite, $p_{c}$ is the vector of dynamic model parameters including atmosphere drag coefficient and light pressure coefficient and coefficients of experiential acceleration and others, $p_{l}$ is other to be estimated parameters of other model.

Orbit state at any time can be get by integral the dynamic model(1), so the parameters to be estimated at time $t$ can be transformed to initial time $t_{0}$, noted as $X_{0}=\left(r_{0}, \dot{r}_{0}, p_{c}, p_{l}\right)^{\mathrm{T}}$, then the observation function can be described as

$$
H=G\left(X_{0}, b\right)+\varepsilon
$$

Where , $b$ is the parameter with no relation to dynamic model, such as index of system error.

In the JPL/ODP and GTDS, there are some parts of parameters meaning considering vector, which is known with low precision. Dividing the all parameters to two parts, named estimating vector $\chi$ and considering vector $z$, and dividing the estimating vector $\chi$ to dynamic model parameters $X_{0}$ and observation model parameters $b$. The observation function can be rewritten as

$$
H=G(\chi, z)+\varepsilon
$$

Let the transcendental value of estimating vector $\chi$ and considering vector $z$ be $\chi_{0}, z_{0}$.

Where, $\mathrm{E}\left(\chi_{0}\right)=\chi, \mathrm{E}\left(z_{0}\right)=z, \operatorname{cov}\left(\chi_{0}\right)=P_{\chi 0}, \operatorname{cov}\left(z_{0}\right)=P_{z 0}$.

Define 1: suppose the loss function be,

$$
\tilde{Q}(\chi)=\left(H-G\left(\chi, z_{0}\right)\right)^{\mathrm{T}} W\left(H-G\left(\chi, z_{0}\right)\right)^{\mathrm{T}}+\left(\chi-\chi_{0}\right)^{\mathrm{T}} P_{\chi}^{-1}\left(\chi-\chi_{0}\right)
$$

Where $W$ is weight matrix of observation.

The second item of the right parts in the above formula(4) is to restrict the estimating value to the transcendental value with the covariance matrix $P_{\chi}$. Based on the iterative method of Gauss-Newton, unwrap the observation function at $\chi_{i}$,

$$
G(\chi, z)=G\left(\chi_{i}, z_{0}\right)+B_{1} \Delta \chi_{i}+B_{2} \Delta z_{i}
$$

Where, $\Delta \chi_{i}=\chi-\chi_{i}, \Delta z_{i}=z-z_{0}, B_{1}=\left.\left(\frac{\partial G}{\partial \chi}\right)\right|_{\chi=\chi_{i}, z=z_{0}}, B_{2}=\left.\left(\frac{\partial G}{\partial z}\right)\right|_{\chi=\chi_{i}, z=z_{0}}$.

The linear loss function can be obtain when put the observation function (5)to loss function(4),

$$
Q\left(\Delta \chi_{i}\right)=\left(\Delta H_{i}-B_{1 i} \Delta \chi_{i}\right)^{\mathrm{T}} W\left(\Delta H_{i}-B_{1 i} \Delta \chi_{i}\right)+\left(\Delta \chi_{i}-\Delta \tilde{\chi}_{i}\right)^{\mathrm{T}} P_{\chi}^{-1}\left(\Delta \chi_{i}-\Delta \tilde{\chi}_{i}\right)
$$

Where, $\Delta H_{i}=H-G\left(\chi_{i}, z\right)$ is the residual error of calculation measurement data and actual measurement data, named OC residual error. $\Delta \tilde{\chi}_{i}=\chi_{0}-\chi_{i}$ is the error of transcendental value and iterative value at $i$. 
Based on the least square estimation theory, the value of $\Delta \chi_{i}$ to minimize Q,

$$
\Delta \hat{\chi}_{i+1}=\left(B_{1 i}^{\mathrm{T}} W B_{1 i}+P_{\chi}^{-1}\right)^{-1}\left(B_{1 i}^{\mathrm{T}} W \Delta H_{i}+P_{\chi}^{-1} \Delta \tilde{\chi}_{i}\right)
$$

Then the estimation is,

$$
\hat{\chi}_{i+1}=\chi_{0}+\sum_{k=1}^{i+1} \Delta \hat{\chi}_{k}=\hat{\chi}_{i}+\Delta \hat{\chi}_{i+1}
$$

The orbit determination method is different with different method to solve the dynamic model and state transfer matrix.

\subsection{Orbit determination based on analytical method}

In analytical method, the ephemeris value can be calculated by mean elements, simple and with high efficiency. And it ignore up two rank chronically items and all the periodic items, for the degree of all the items is $O\left(J_{2}\right)$, with conditions of $n\left(t-t_{0}\right)=1 / J_{2}$.

The partial differential coefficient matrix of observation vector to dynamic model parameters can be got by chain principle,

$$
B=\frac{\partial G}{\partial(r, \dot{r})} \cdot \frac{\partial(r, \dot{r})}{\partial X} \cdot \frac{\partial X}{\partial X_{0}}
$$

1. the first kind partial differential coefficient matrix $\frac{\partial G}{\partial(r, \dot{r})}$

The sensitive degree of observation value at time $t$ to position and velocity vector is reflected by the first kind partial differential coefficient matrix

2. the second kind partial differential coefficient matrix $\frac{\partial(r, \dot{r})}{\partial X}$

The ephemeris of satellite and the position and velocity can be transformed each other, so the second kind partial differential coefficient is transfer matrix.

3. the third kind partial differential coefficient matrix $\frac{\partial X}{\partial X_{0}}$

The third kind partial differential coefficient matrix is the state transfer matrix, it shows the transform from vector at time $t$ to initial time. In the process of orbit determination based on analytical method, the matrix can be predigested.

\subsection{Orbit determination based on numerical method}

Although the dynamic model can be solved by analytical method, it generally cannot obtain the exact value, for the complicated dynamic model. With numerical method, the position and velocity of satellite at time t obtained by integral. The formula (1) can transform as 


$$
\begin{gathered}
\frac{\partial \ddot{r}}{\partial X_{0}}=\frac{\partial \ddot{r}}{\partial r} \frac{\partial r}{\partial X_{0}}+\frac{\partial \ddot{r}}{\partial \dot{r}} \frac{\partial \dot{r}}{\partial X_{0}}+\left(\frac{\partial \ddot{r}}{\partial X_{0}}\right)_{e} \\
\frac{\mathrm{d}^{2}}{\mathrm{~d} t^{2}}\left(\frac{\partial r}{\partial X_{0}}\right)=\frac{\partial \ddot{r}}{\partial r} \frac{\partial r}{\partial X_{0}}+\frac{\partial \ddot{r}}{\partial \dot{r}} \frac{\mathrm{d}}{\mathrm{d} t}\left(\frac{\partial r}{\partial X_{0}}\right)+\left(\frac{\partial \ddot{r}}{\partial X_{0}}\right)_{e} \\
A(t)=\frac{\partial \ddot{r}(t)}{\partial r}, C(t)=\frac{\partial \ddot{r}(t)}{\partial X_{0}}, B(t)=\frac{\partial \ddot{r}(t)}{\partial \dot{r}}, Y(t)=\frac{\partial r(t)}{\partial X_{0}} \\
\ddot{Y}=A(t) Y+B(t) \dot{Y}+C(t)
\end{gathered}
$$

Where $Y$ is state transfer matrix.

The value of formula (10) can be get by Adams-Cowell integral method.

\subsection{Orbit determination based on difference method}

Let the orbit determination be

$$
\left\{\begin{array}{l}
\dot{X}(t)=f(X(t), t) \\
X\left(t_{0}\right)=X_{0}
\end{array}\right.
$$

Where, $X(t)=\left(r, \dot{r}, p_{c}\right)^{\mathrm{T}}$ is orbit state at time $\mathrm{t},(r, \dot{r})=F\left(r_{0}, \dot{r}_{0}, p_{c}\right)$.

Let the dynamic model parameters are,

$$
p_{c}=\left(C_{D 1}, \cdots C_{D n_{1}} ; C_{R 1}, \cdots, C_{R n_{2}} ; C_{E 1}, \cdots, C_{E n 3}\right)
$$

where $C_{D i}\left(i=1,2, \cdots, n_{1}\right)$ is the atmosphere coefficients of $i$ th zone, $C_{R i}\left(i=1,2, \cdots, n_{2}\right)$ is the sunlight pressure coefficient of $i$ th zone, $C_{E i}\left(i=1,2, \cdots, n_{3}\right)$ is experiential acceleration coefficient of $i$ th zone. The total number of parameters to be estimated is $m=6+n_{1}+n_{2}+n_{3}$, then the state transfer matrix is,

$$
\frac{\partial(r, \dot{r})}{\partial\left(r_{0}, \dot{r}_{0}, p_{c}\right)}=\frac{\partial F\left(r_{0}, \dot{r}_{0}, t_{0}, t, p_{c}\right)}{\partial\left(r_{0}, \dot{r}_{0}, p_{c}\right)}=\left(\begin{array}{ccccccccccc}
\frac{\partial x}{\partial x_{0}} & \frac{\partial x}{\partial y_{0}} & \cdots & \frac{\partial x}{\partial v_{z 0}} & \cdots & \frac{\partial x}{\partial C_{D i}} & \cdots & \frac{\partial x}{\partial C_{R i}} & \cdots & \frac{\partial x}{\partial C_{E n_{3}}} \\
\frac{\partial y}{\partial x_{0}} & \frac{\partial y}{\partial y_{0}} & \cdots & \frac{\partial y}{\partial v_{z 0}} & \cdots & \frac{\partial y}{\partial C_{D i}} & \cdots & \frac{\partial y}{\partial C_{R i}} & & \frac{\partial x}{\partial C_{E n_{3}}} \\
\vdots & \vdots & & \vdots & & \vdots & & \vdots & \ddots & \vdots \\
\frac{\partial v_{z}}{\partial x_{0}} & \frac{\partial v_{z}}{\partial y_{0}} & \cdots & \frac{\partial v_{z}}{\partial v_{z 0}} & \cdots & \frac{\partial v_{z}}{\partial C_{D i}} & \cdots & \frac{\partial v_{z}}{\partial C_{R i}} & \cdots & \frac{\partial v_{z}}{\partial C_{E n_{3}}}
\end{array}\right)_{6 \times m}
$$

Where, $\frac{\partial \Upsilon}{\partial \Theta}\left(\Upsilon=x, y, \cdots v_{z}, \Theta=x_{0}, y_{0}, \cdots, C_{D i}, \cdots, C_{R i}, \cdots, C_{E i}, \cdots\right)$ is effect of the initial state $\Theta$ to state $\Upsilon$ at time $t$, so the value can be simply got, 


$$
\frac{\partial \Upsilon}{\partial \Theta}=\frac{\Upsilon(\Theta+\Delta)-\Upsilon(\Theta)}{\Delta}
$$

Where, $\Delta$ is little value, $\Upsilon(\Theta+\Delta)$ is the state calculated by the initial state $\Theta$ add $\Delta$.

The other more concise method based on difference is directly to deal with the Jacobi matrix

$$
B=\frac{\partial G}{\partial\left(r_{0}, \dot{r}_{0}, p_{c}\right)}=\left(\begin{array}{cccccccccc}
\frac{\partial h_{1}}{\partial x_{0}} & \frac{\partial h_{1}}{\partial y_{0}} & \cdots & \frac{\partial h_{1}}{\partial v_{z 0}} & \cdots & \frac{\partial h_{1}}{\partial C_{D i}} & \cdots & \frac{\partial h_{1}}{\partial C_{R i}} & \cdots & \frac{\partial h_{1}}{\partial C_{E n_{3}}} \\
\frac{\partial h_{2}}{\partial x_{0}} & \frac{\partial h_{2}}{\partial y_{0}} & \cdots & \frac{\partial h_{2}}{\partial v_{z 0}} & \cdots & \frac{\partial h_{2}}{\partial C_{D i}} & \cdots & \frac{\partial h_{2}}{\partial C_{R i}} & & \frac{\partial h_{2}}{\partial C_{E n_{3}}} \\
\vdots & \vdots & & \vdots & & \vdots & & \vdots & \ddots & \vdots \\
\frac{\partial h_{n_{k}}}{\partial x_{0}} & \frac{\partial h_{n_{k}}}{\partial y_{0}} & \cdots & \frac{\partial h_{n_{k}}}{\partial v_{z 0}} & \cdots & \frac{\partial h_{n_{k}}}{\partial C_{D i}} & \cdots & \frac{\partial h_{n_{k}}}{\partial C_{R i}} & \cdots & \frac{\partial h_{n_{k}}}{\partial C_{E n_{3}}}
\end{array}\right)_{n_{k} \times m}
$$

Where, $n_{k}$ is the number of observation elements.

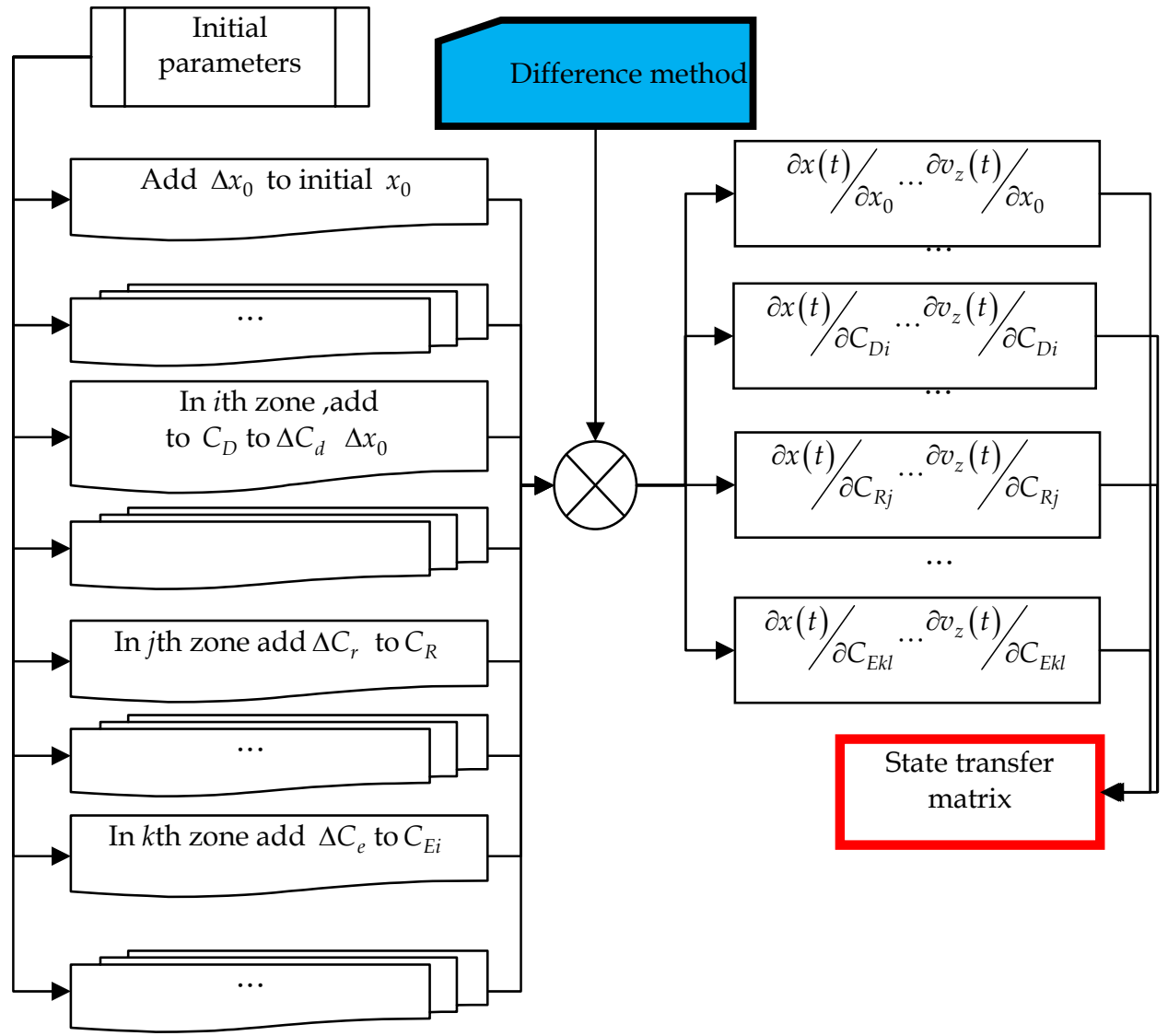

Fig. 2. Flow chart of orbit determination based on difference method. 


\section{The uniform method of orbit determination with uncertainty model error}

The satellite dynamic model can be descriped more and more subtle, but the actual force still can not be entirely expressed by the model. There are some unknown or inexplicable perturbation force and modled pertubation force with unkonwn parameter, for example, the atmosphere consistency parameter in the atmosphere pertubation force modle. So the model error of satellite dynamic model is the inevitable fact. If the orbit determination with no model error compensation is processed, the degree of orbit error will be equal with the degree of dynamic model error, see figure 3.

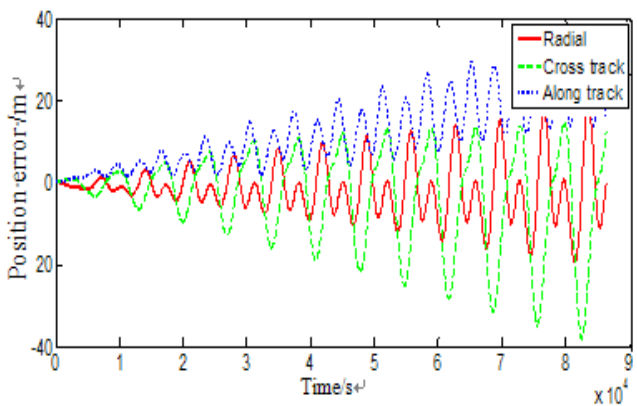

(a) Model error

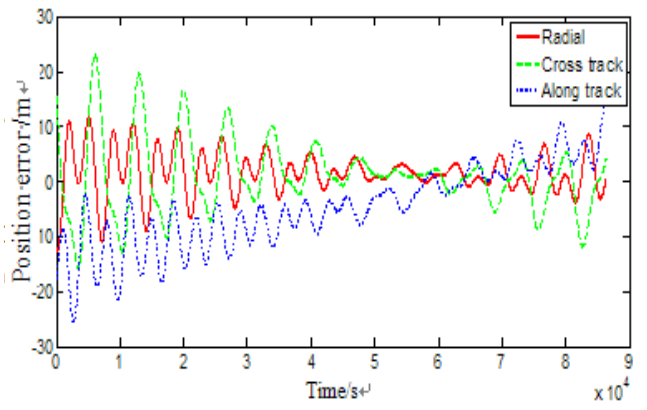

(b) Orbit determination error

Fig. 3. Modle error and orbit determination error with no model compensation.

The dynamic model in the J2000 reference frame can be described as the following:

$$
\left\{\begin{array}{l}
\dot{r}=F(r, t)+F_{\varepsilon}(r, t) \\
r\left(t_{0}\right)=r_{0}
\end{array}\right.
$$

where $r=\left(x, y, z, v_{x}, v_{y}, v_{z}\right)^{T}$ is the satellite state vector, $F_{\varepsilon}(r, t)$ is dynamic model error.

The formal function of state transfer can be described as

$$
\left\{\begin{array}{l}
\dot{\Phi}\left(t, t_{0}\right)=A(t) \Phi\left(t, t_{0}\right) \\
\Phi\left(t_{0}, t_{0}\right)=I
\end{array}\right.
$$

Note, $X_{0}=\left(r_{0}, \dot{r}_{0}, p_{c}\right)^{\mathrm{T}}$. Then the formula of dynamic model can be showed as the following,

$$
\begin{gathered}
\frac{\mathrm{d}^{2}}{\mathrm{~d} t^{2}}\left(\frac{\partial r}{\partial X_{0}}\right)=\frac{\partial \ddot{r}}{\partial r} \frac{\partial r}{\partial X_{0}}+\frac{\partial \ddot{r}}{\partial \dot{r}} \frac{\mathrm{d}}{\mathrm{d} t}\left(\frac{\partial r}{\partial X_{0}}\right)+\left(\frac{\partial \ddot{r}}{\partial X_{0}}\right)_{e} \\
A(t)=\frac{\partial \ddot{r}(t)}{\partial r}, C(t)=\frac{\partial \ddot{r}(t)}{\partial X_{0}}, B(t)=\frac{\partial \ddot{r}(t)}{\partial \dot{r}}, Y(t)=\frac{\partial r(t)}{\partial X_{0}}
\end{gathered}
$$

Where, $A(t)=\frac{\partial \ddot{r}}{\partial r}=\frac{\partial\left(F+F_{\varepsilon}\right)}{\partial r} \triangleq A_{0}(t)+A_{\varepsilon}(t)$. 
Based on the differential equation theory, the form root of state transfer function (15) is

$$
\Phi=C e^{\left(A_{0}+A_{\varepsilon}\right)}=C e^{\left(A_{0}\right)} \cdot C e^{\left(A_{\varepsilon}\right)}=\Phi_{0} \Phi_{\varepsilon}
$$

Where, $\Phi_{0}$ is the state transfer matrix of perturbation $F$ with known model, while $\Phi_{\varepsilon}$ is the state transfer matrix of $F_{\varepsilon}$

The orbit function can be deployed as:

$$
\dot{r}(t)=F(r, t)=F\left(r^{*}, t\right)+\left.\left(\frac{\partial F+F_{\varepsilon}}{\partial r}\right)\right|_{r=r^{*}} \Delta r+F_{\varepsilon}\left(r^{*}, t\right) \cdots
$$

let $\Delta r(t)=r(t)-r^{*}(t)$,

$$
A(t)+A_{\varepsilon}(t)=\left.\left(\frac{\partial\left(F+F_{\varepsilon}\right)}{\partial r}\right)\right|_{r=r^{*}}
$$

The formula(17)can be noted:

$$
\Delta \dot{r}(t)=\left(A(t)+A_{\varepsilon}(t)\right) \Delta r(t)
$$

The formula (18) can be solved by the differential equations theory, which can be expressed as following:

$$
\Delta r(t)=\left(\Phi\left(t, t_{0}\right)+\Phi_{\varepsilon}\left(t, t_{0}\right)\right) \Delta r\left(t_{0}\right)
$$

Where $\Phi\left(t, t_{0}\right)$ is state transferring matrix toward precise parameter perturbation force, and $\Phi_{\varepsilon}\left(t, t_{0}\right)$ is state transferring matrix toward perturbation force error.

\subsection{Mesasure function with on system error}

The measure function can be noted as the following,

$$
H=\tilde{G}\left(r, \dot{r}, p_{c}, t\right)+\varepsilon=G\left(X_{0}, t_{0}, t\right)+\varepsilon, X_{0}=\left(r_{0}, \dot{r}_{0}, p_{c}\right)^{\mathrm{T}}
$$

Where, the relation of satellite position and velocity at time $t$ and other to be estimated parameters is described by the first equation, and the second equation shows the relation at initial time $t_{0}$.

Then, the orbit function with uncertainty dynamic model error can be described as,

$$
\left\{\begin{array}{l}
\dot{X}=f(X, t)+f_{\varepsilon} \\
H=G\left(X_{0}, t_{0}, t\right)+\varepsilon
\end{array}\right.
$$

Where, $f_{\varepsilon}=\left(0, F_{\varepsilon}, 0\right)^{\mathrm{T}}$.

Based on the differential coefficient improve process, we can get 


$$
H-G\left(X_{0}^{*}, t_{0}, t\right)=\frac{\partial G\left(X_{0}^{*}, t_{0}, t\right)}{\partial X_{0}} \Delta X_{0}+\varepsilon=B \Delta X_{0}+\varepsilon
$$

Note,

$$
Y=B \Delta X_{0}+\varepsilon
$$

Where, $Y$ is OC residual error, $B=\frac{\partial G}{\partial\left(r_{c}, \dot{r}_{c}\right)} \cdot \frac{\partial\left(r_{c}, \dot{r}_{c}\right)}{\partial(r, \dot{r})} \cdot \frac{\partial(r, \dot{r})}{\partial X_{0}}, \varepsilon$ is error vector.

Based on the formula(20), the matrix satellite state transfer is: $\Phi=\Phi_{0} \Phi_{\varepsilon}$, then

$$
\begin{gathered}
\tilde{B}=\frac{\partial G}{\partial\left(r_{c}, \dot{r}_{c}\right)} \cdot \frac{\partial\left(r_{c}, \dot{r}_{c}\right)}{\partial(r, \dot{r})} \cdot\left[\Phi, \frac{\partial(r, \dot{r})}{\partial p_{c}}\right] \\
=\frac{\partial G}{\partial\left(r_{c}, \dot{r}_{c}\right)} \cdot \frac{\partial\left(r_{c}, \dot{r}_{c}\right)}{\partial(r, \dot{r})} \cdot\left[\Phi_{0} \Phi_{\varepsilon}, \frac{\partial(r, \dot{r})}{\partial p_{c}}\right] \\
Y=H-G\left(X_{0}^{*}, t_{0}, t\right)=H-\tilde{G}\left(\Phi X_{0}^{*}, t\right)=H-\tilde{G}\left(\Phi_{0} \Phi_{\varepsilon} X_{0}^{*}, t\right)
\end{gathered}
$$

In fact the matrix $\Phi_{\varepsilon}$ is unkown, So formula (21) should be designed as

$$
Y=H-\tilde{G}\left(\Phi_{0} X_{0}^{*}, t\right)-S
$$

Where $S=\tilde{G}\left(\Phi_{0} \Phi_{\varepsilon} X_{0}^{*}, t\right)-\tilde{G}\left(\Phi_{0} X_{0}^{*}, t\right)$, expressed the error caused by dynamic model error. The error $S$ is uncertainty for the unknown matrix $\Phi_{\varepsilon}$.

So the orbit determination function (22) should be changed as

$$
Y=\tilde{B} \Delta X_{0}+S+\varepsilon
$$

For the low precision need of $B$ matrix and the minuteness value of $\Phi_{\varepsilon}$, the formula (23) can be changed as the following,

$$
Y=B \Delta X_{0}+S+\varepsilon
$$

The observation system error $S_{d}$ caused by dynamic model error can be decomposed from uncertainty model error $S$.Then,

$$
\begin{gathered}
S_{d}=\tilde{G}\left(\Phi_{0} \Phi_{\varepsilon} X_{0}^{*}, t\right)-\tilde{G}\left(\Phi_{0} X_{0}^{*}, t\right) \\
\tilde{G}\left(\Phi_{0} \Phi_{\varepsilon} X_{0}^{*}, t\right)=\tilde{G}\left(\Phi_{0}\left(\Phi_{\varepsilon}-I\right) X_{0}^{*}+\Phi_{0} X_{0}^{*}, t\right)
\end{gathered}
$$

Unwrap the above formula (25) at $\Phi_{0} X_{0}^{*}$ 


$$
\begin{gathered}
\tilde{G}\left(\Phi_{0} \Phi_{\varepsilon} X_{0}^{*}, t\right)=\tilde{G}\left(\Phi_{0}\left(\Phi_{\varepsilon}-I\right) X_{0}^{*}+\Phi_{0} X_{0}^{*}, t\right) \\
=\tilde{G}\left(\Phi_{0} X_{0}^{*}, t\right)+\frac{\partial \tilde{G}}{\partial \Phi_{0} X_{0}^{*}} \Phi_{0}\left(\Phi_{\varepsilon}-I\right) X_{0}^{*} \\
S_{d}=\frac{\partial \tilde{G}}{\partial \Phi_{0} X_{0}^{*}} \Phi_{0}\left(\Phi_{\varepsilon}-I\right) X_{0}^{*} \\
S_{d}+\frac{\partial \tilde{G}}{\partial \Phi_{0} X_{0}^{*}} \Phi_{0} X_{0}^{*}=\frac{\partial \tilde{G}}{\partial \Phi_{0} X_{0}^{*}} \Phi_{0} \Phi_{\varepsilon} X_{0}^{*}
\end{gathered}
$$

In fact, if there are no any dynamic model error, then $\Phi_{\varepsilon}=I S_{d}=0$.

$$
\begin{aligned}
& \left(\begin{array}{c}
S_{d 1} \\
S_{d 2} \\
\vdots \\
S_{d n}
\end{array}\right)+\left(\begin{array}{cccc}
\frac{\partial \tilde{G}_{1}}{\partial r\left(t_{1}\right)} & & & \\
& \frac{\partial \tilde{G}_{2}}{\partial r\left(t_{2}\right)} & & \\
& & \ddots & \\
& & & \frac{\partial \tilde{G}_{n}}{\partial r\left(t_{n}\right)}
\end{array}\right)\left(\begin{array}{c}
\Phi_{0}\left(t_{0}, t_{1}\right) \\
\Phi_{0}\left(t_{0}, t_{2}\right) \\
\vdots \\
\Phi_{0}\left(t_{0}, t_{n}\right)
\end{array}\right) X_{0}^{*}
\end{aligned}
$$

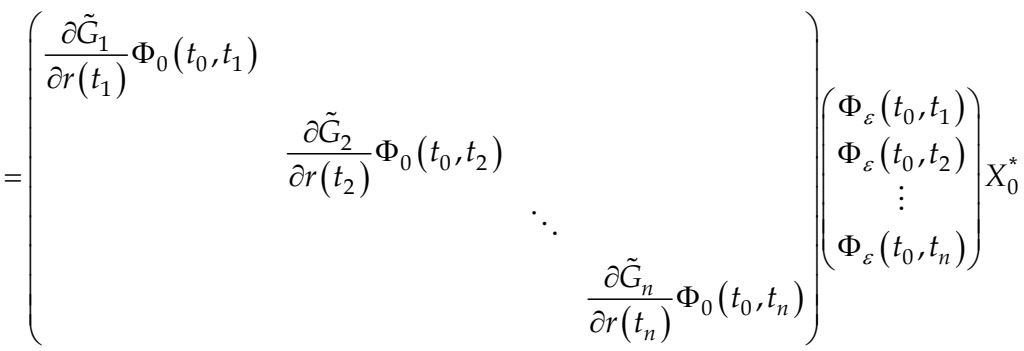

Form the matrix formula function (27), it can be get that $S_{d i}=S\left(t_{d i}\right),(i=1,2, \cdots, n)$ is the model error value estimated by semi-linear model orbit determination, $\frac{\partial G_{i}}{r_{i}}$ is the differential coefficient of observation function to satellite state at $t_{i}, \Phi_{0}$ is the state transfer matrix of known perturbation force. So only the $\Phi_{\varepsilon}\left(t_{i}, t_{0}\right)$ in matrix function (27)is an unknown quantity. $\Phi_{\varepsilon}\left(t_{i}, t_{0}\right)$ shows the influence of error perturbation force to satellite state, the function(27) cannot be solved because the matrix function contains $n \times 6 \times m$ unknown parameters. There are two methods to solve the problem.

\subsubsection{Fitting the sate transfer matrix by base functions}

Because the $\Phi_{\varepsilon}\left(t_{i}, t_{0}\right)$ can be adapted to dynamic function, then it can be drawn that 


$$
\frac{d^{2}}{d t^{2}} \frac{\partial r_{\varepsilon}(t)}{\partial X_{0}}=\frac{\partial f_{\varepsilon}}{\partial r_{\varepsilon}} \frac{\partial r_{\varepsilon}(t)}{\partial X_{0}}+\frac{\partial f_{\varepsilon}}{\partial \dot{r}_{\varepsilon}} \frac{d}{d t} \frac{\partial r_{\varepsilon}(t)}{\partial X_{0}}+\frac{\partial f_{\varepsilon}}{\partial X_{0}}
$$

Where, $r_{\varepsilon}$ is the position vector effected by perturbation force error $f_{\varepsilon}$.

So the element in matrix $\frac{\partial r_{\varepsilon}(t)}{\partial X_{0}}$ is consecutive and slippery, and can be approached with limited base functions. Let the base functions $\mathrm{b} \sum_{i=1}^{\lambda} a_{i} B_{i}(t)$, and the $\lambda$ is the estimated number of base function, $a_{i}, B_{i}$ are coefficients of base functions and base functions.

$$
\begin{gathered}
S_{d i}+\frac{\partial \tilde{G}_{i}}{\partial r\left(t_{i}\right)} \Phi_{0}\left(t_{0}, t_{i}\right) X_{0}^{*}=\frac{\partial \tilde{G}_{i}}{\partial r\left(t_{i}\right)} \Phi_{0}\left(t_{0}, t_{i}\right) \hat{\Phi}_{\varepsilon} X_{0}^{*} \\
\hat{\Phi}_{\varepsilon}=\left(\begin{array}{lll}
\sum_{j=1}^{\lambda} a_{j_{11}} B_{j}\left(t_{i}\right) & \sum_{j=1}^{\lambda} a_{j_{12}} B_{j}\left(t_{i}\right) & \sum_{j=1}^{\lambda} a_{j_{1 m}} B_{j}\left(t_{i}\right) \\
\sum_{j=1}^{\lambda} a_{j_{21}} B_{j}\left(t_{i}\right) & \sum_{j=1}^{\lambda} a_{j_{22}} B_{j}\left(t_{i}\right) & \sum_{j=1}^{\lambda} a_{j_{2 m}} B_{j}\left(t_{i}\right) \\
\sum_{j=1}^{\lambda} a_{j_{61}} B_{j}\left(t_{i}\right) & \sum_{j=1}^{\lambda} a_{j_{62}} B_{j}\left(t_{i}\right) & \sum_{j=1}^{\lambda} a_{j_{6 m}} B_{j}\left(t_{i}\right)
\end{array}\right)_{6 \times m}
\end{gathered}
$$

There are $6 \times m \times \lambda$ parameters in the formula (29), so when $n \geq 6 \times m \times \lambda$, the form of state transfer of dynamic model error can be solved.

\subsubsection{Method of orbit state}

It is easy to be matrix singularity for to estimated parameters, if the state transfer matrix error is solved directly by formula (27). There is other method to solve the problem to get the orbit error $\Delta X$ based on the above formula (26).

$$
S_{d}=\frac{\partial \tilde{G}}{\partial \Phi_{0} X_{0}^{*}} \Delta X
$$

Then, the same fitting method can be used to the orbit error $\Delta X$, and get the right value with only $6 \times \lambda$ parameters.

\subsection{Orbit determination function with uncertainty observation model error}

The uncertainty observation model error mains the error of unable to model and modifying residual error with no form. With the more and more complicated observation, the system error will be more and more intricate. If no correct disposal method, the system observation error will mix into the observation data, and to guide the wrong direction. 
There are two kinds of uncertainty observation error, one is residual error, such as atmosphere modify error and residual error of measure system error of radar. All of the form can be noted as

$$
H=\tilde{G}\left(r, \dot{r}, p_{c}, t\right)+S+\varepsilon=G\left(X_{0}, t_{0}, t\right)+S+\varepsilon
$$

The other is occurred in elements of measurement data, it can be caused by any error of measurement parameters, such as position error of station.

$$
H=\tilde{G}\left(r, \dot{r}, p_{c}, t, S\right)+\varepsilon
$$

In fact the above formula (32) can be noted as the formula (31), and then the uncertainty observation orbit determination function can be described as the following,

$$
\begin{gathered}
\left\{\begin{array}{l}
\dot{X}=f(X, t) \\
H=G\left(X_{0}, t_{0}, t\right)+S+\varepsilon
\end{array}\right. \\
Y=B \Delta X_{0}+S+\varepsilon
\end{gathered}
$$

\subsection{Uniform format of orbit determination semi-linear model}

When there are uncertainty model error both the observation model and the dynamic model, the orbit determination function can be drawn as,

$$
\left\{\begin{array}{l}
\dot{X}=f(X, t)+f_{\varepsilon} \\
H=G\left(X_{0}, t_{0}, t\right)+g_{\varepsilon}+\varepsilon
\end{array}\right.
$$

The satellite transfer matrix function is $\Phi=\Phi_{0} \Phi_{\varepsilon}$, then

$$
Y=H-G\left(X_{0}^{*}, t_{0}, t\right)-g_{\varepsilon}=H-\tilde{G}\left(\Phi X_{0}^{*}, t\right)-g_{\varepsilon}=H-\tilde{G}\left(\Phi_{0} \Phi_{\varepsilon} X_{0}^{*}, t\right)-g_{\varepsilon}
$$

For the uncertainty of matrix $\Phi_{\varepsilon}$, the actual model of formula (33) can be expressed as

$$
Y=H-\tilde{G}\left(\Phi_{0} X_{0}^{*}, t\right)-S-g_{\varepsilon}
$$

Where $S=\tilde{G}\left(\Phi_{0} \Phi_{\varepsilon} X_{0}^{*}, t\right)-\tilde{G}\left(\Phi_{0} X_{0}^{*}, t\right)$ is the error caused by dynamic model error mixed in observation model. The uniform can be get, because the value $S$ and $g_{\varepsilon}$ are the same kind of uncertainty model error.

The uniform formula function of orbit determination with uncertainty model in dynamic model and observation model can be shown as,

$$
Y=B \Delta X_{0}+S+\varepsilon
$$

Although the uncertainty error $S$ is uniform, it is different in the character. For example, the form of dynamic model error is generally periodic with given frequency. 


\section{The solve method of uniform orbit determination with uncertainty model error based on semi-parametric model}

\subsection{Semi-parametric model}

Let $b_{1}, b_{2}, \cdots, b_{p}$ and $t_{1}, t_{2}, \cdots, t_{q}$ be the variable array of $L$, and $b_{1}, b_{2}, \cdots, b_{p}$ are main part with linear character to $L$, and $t_{1}, t_{2}, \cdots, t_{q}$ can be explained as disturbed factors with non linear property, so the semi-parametric model will be noted as,

$$
L_{i}=b_{i}^{T} x+s\left(t_{i}\right)+\varepsilon_{i}, \quad i=1,2, \cdots, n
$$

Where $b_{i}=\left(b_{i 1}, b_{i 2}, \cdots, b_{i d}\right)^{T}, x=\left(x_{1}, x_{2}, \cdots, x_{d}\right)^{T}, x$ is the parameters to be estimated, $\varepsilon_{i}$ is the stochastic error.

\subsection{Two stage parametric estimator}

It should be noticed that nonparametric component $g(t)$ contains not only dynamic model error, but also measurement system error and so on, nonparametric component $g(t)$ cannot be described by parameters, so two stage estimator can be applied to solve $g(t)$.

Suppose $E(g(t))=\alpha, v=g(t)-\alpha+\varepsilon(t)$, the error function can be noted as:

$$
v(t)=B \cdot \Delta r+I \cdot \alpha-h
$$

To construct the function based on Lagrange Method,

$$
\Phi=v^{T} v+2 \lambda^{T}(B \Delta r+I \alpha-h-v)
$$

Calculate the minimum value of formula (37), then the first stage estimation of $\Delta r$ and $\alpha$ can be described as,

$$
\begin{gathered}
\Delta \hat{r}_{1}=\left(B^{T} B\right)^{-1} B^{T}\left(h-I \hat{\alpha}_{1}\right) \\
\hat{\alpha}_{1}=\left(I^{T} I\right)^{-1} I^{T}\left(h-B \Delta \hat{r}_{1}\right) \\
S(t)=h-B \cdot \Delta \hat{r}_{1}
\end{gathered}
$$

Formula (40)can be estimated with kernel estimator,

$$
\hat{g}(t)=\frac{\sum_{i=1}^{n} S_{i} W_{i n}(x)}{\sum_{i=1}^{n} W_{i n}(x)}
$$

Where, $W_{\text {in }}(x)$ is kernel weight function. The common kernel weight function is NadarayaWatson (41), and Gasser-Müller(42), 


$$
\begin{gathered}
W_{i n}(x)=\frac{K\left(\frac{t-t_{i}}{h}\right)}{\sum_{i=1}^{n} K\left(\frac{t-t_{i}}{h}\right)} \\
W_{i n}(x)=\frac{1}{h} \int_{T_{i-1}}^{T_{i}} K\left(\frac{t-u}{h}\right) d u
\end{gathered}
$$

Where $T_{0}=t_{0}, T_{i}=\left(t_{i}+t_{i+1} / 2\right), h$ is the window to be set.

\subsection{Stahel-Donoho kernel estimator based on data depth}

For the influence of stochastic error, the nonparametric component contains not only system error but also stochastic error, so the information and reliability of different sampling measurement data are different, and so as the weight of observation data. Data depth can describe the degree of every data in the swatch. Many scholar put forward different data depth function based on different requirement. Though defines of data depth are different, the basic idea is the same, which can be shown that the values of data depth are big near the middle of data, on the contrary, the values are small far from the middle.

Define the data depth of $\varepsilon$ with distribution $F$ based on the basic idea [6],

$$
D(\varepsilon, F)=\frac{1}{1+O(S)^{2}}
$$

Where, $O(S)=|\varepsilon-\operatorname{Med}(F)| / M A D(F), \operatorname{Med}(F)$ is median of $\mathrm{F}, M A D(F)$ is median of $|\varepsilon-\operatorname{Med}(F)|$.

The depth $D_{i}$ describes the degree of data $S_{i}$ in the total data.

The common weight function are as following:

$$
\omega(s)=\left\{\begin{array}{l}
\frac{\exp \left(-k\left(1-\frac{s}{\operatorname{med}(D(Z, F))}\right)^{2}\right)-\exp (-k)}{1-\exp (-k)}, s<\operatorname{med}(D(Z, F)) \\
1, s \geq \operatorname{med}(D(Z, F))
\end{array}\right.
$$

Where, $k$ is constant parameter, and med $(D(Z, F))$ is median of depth set.

Then the nonparametric component estimate can be noted as:

$$
\hat{g}(t)=\frac{\sum_{i=1}^{n} S_{i} \omega_{i} W_{i n}(x)}{\sum_{i=1}^{n} \omega_{i} W_{i n}(x)}
$$


Then the nonparametric parts had been estimated with data depth weighted kernel method, put $\hat{g}(t)$ into formula (40), the second estimation of $\Delta r(t)$ will be calculated. The nonparamatric regression can be called Stahel-Donoho Kernel Estimator (SDKE).

\subsection{Simulation experiments}

\subsubsection{Simulation conditions}

The TLE of LEO satellite is as the following:

\section{COSMOS2221}

1 22236U 92080A 08107.80786870.00000150 00000-0 15532-4 01936

222236082.5088327 .45930016739264 .6279095 .301814 .831301358342996

\subsubsection{Simulation results and analysis}

The results of orbit determination based on different methods are printed in figure 5 and figure 6. Numeric results are given in table 1.

\begin{tabular}{|c|c|c|c|}
\hline method & Radial/m & Cross track/m & Along track/m \\
\hline Traditional method & 420.3 & 533.4 & 504.4 \\
\hline $\begin{array}{c}\text { Stahel-Donoho } \\
\text { Kernel Estimator }\end{array}$ & 120.68 & 164.8 & 150.8 \\
\hline
\end{tabular}

Table 1. Orbti determination results of different methods.

In figure1, random error and system error are included with max swing 30 arcsec. In traditional orbit determination method, the ME is treated as white noise, the results of orbit determination based on traditional method was shown in figure 2. It can be seen that the OC residual error still contained the $\mathrm{ME}$ (see in figure 4), while the OC residual error based on SDKE contained no system errors, in another word, OC residual based on SDKE is white noise after model error compensated, so the precision of SDKE was improved largely.

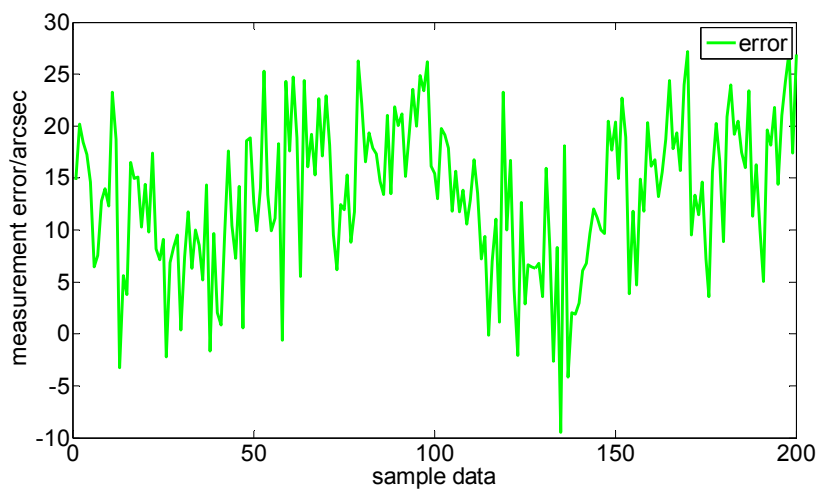

Fig. 4. Measurement data error in simulation experiment. 


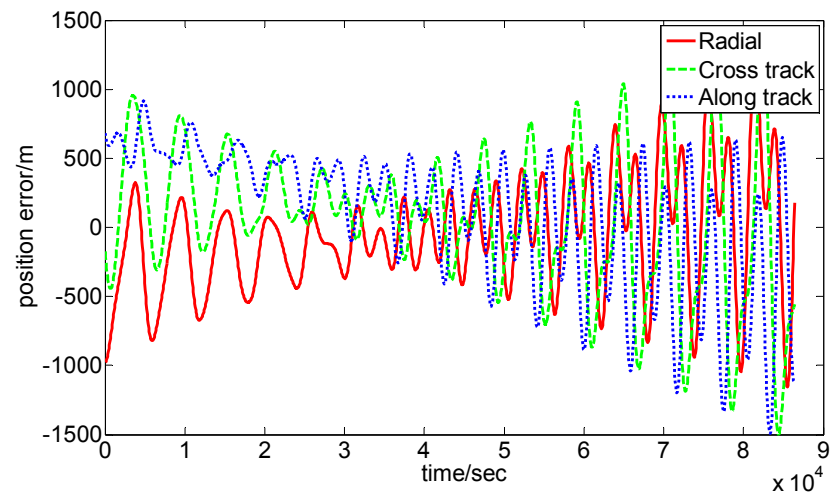

Fig. 5. Results of orbit determination based on traditional method.

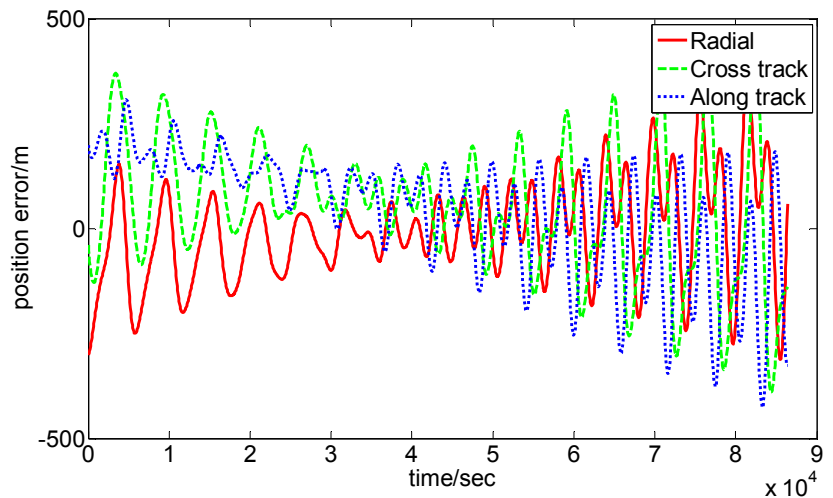

Fig. 6. Results of orbit determination based on SDKE.

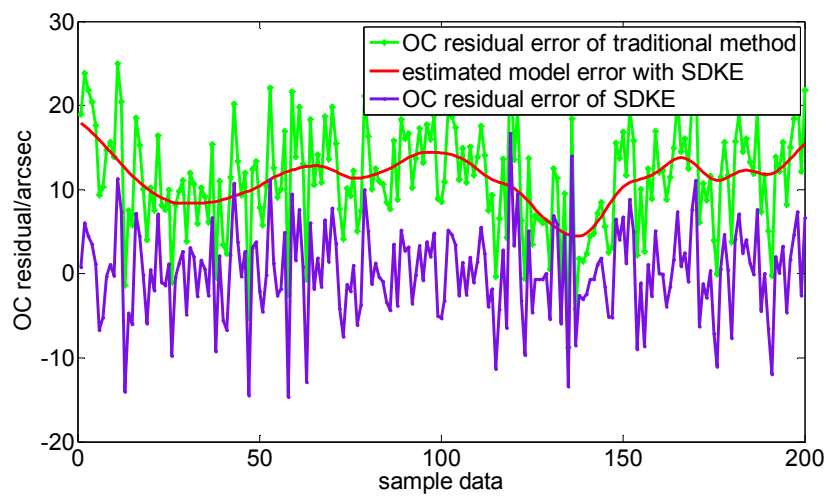

Fig. 7. OC residual of different method.

Model error is the main factor to badly pollute the precision of orbit determination, while semi-parametric is the effective way to compensate the model error. Stahel -Donoho Kernel 
Estimator is with great superiority in robust and efficiency, especially its data depth weight matrix can restrain the gross error in the ME, which will happen usually in space based observation. SDKE method of orbit determination can highly improve the precision of orbit determination in space based surveillance system.

\section{Reduced dynamic orbit determination based on spline method}

The precision of orbit determination depends on the precision of measurement data and the precision of dynamic model. In modern times, the degree of measurement can be limited in millimeter degree, so the key method to improve the precision of orbit determination is to increase the exactness of dynamic model, or to compensate the model error. In the above chapters, some mathematic method are proposed to compensate the uncertainty model error, all those method is focus on the mathematics models, in this chapter, a new orbit determination method based on model error compensation is put forward to deal with directly the dynamic force.

\subsection{Orbit determination based on experiential acceleration}

In order to remove the error caused by dynamic force, many scholars applied the experiential acceleration method. The general form of experiential acceleration is,

$$
f_{R T N}=\left(\begin{array}{c}
C_{R} \cos u+S_{R} \sin u \\
C_{T} \cos u+S_{T} \sin u \\
C_{N} \cos u+S_{N} \sin u
\end{array}\right) \cdot\left(\begin{array}{c}
e_{R} \\
e_{T} \\
e_{N}
\end{array}\right)
$$

where $u$ is earth latitude angle, $e_{R}, e_{T}, e_{N}$ is unit vector of radial, track and cross of satellite.

The experiential acceleration form of (45)is proposed based on the frequency error with 1cycle-per-revolution, and this model can absorb effectively the dynamic model error, and used in the orbit determination of satellite CHAMP. When the form of dynamic model changed, the above model may not be available. So the spline model is proposed to deal with the uncertainty dynamic model error.

Decompose the observation arc into some little arc,

$$
\begin{aligned}
\pi: a=u_{0}<u_{1}<\cdots<u_{N}= & b, u_{i+1}-u_{i}=h, u_{-1}=u_{0}-h, u_{N+1}=u_{N}+h \\
f_{R T N} & =\left(\begin{array}{l}
\sum_{j=-1}^{N+1} d_{R j} \varphi_{j}(u) \\
\sum_{j=-1}^{N+1} d_{T j} \varphi_{j}(u) \\
\sum_{j=-1}^{N+1} d_{N j} \varphi_{j}(u)
\end{array}\right) \cdot\left(\begin{array}{c}
e_{R} \\
e_{T} \\
e_{N}
\end{array}\right)
\end{aligned}
$$

Where, $u$ is earth latitude angle, $\varphi_{j}(u)$ is spline base function in arc, $e_{R}, e_{T}, e_{N}$ is unit vector of radial, track and cross of satellite. 


\subsection{Precision of model based on dynamic model smoothing}

It can be realized by dynamic model smoothing to evaluate the capability of experiential acceleration compensation method. Suppose the actual orbit data be observation data, calculate the orbit parameters by compensation orbit determination. In the following experiment, the actual orbit data is the CHAMP orbit data download by GFZ, and different method of orbit determination will be used to smooth the orbit in orbit to prove the experiential acceleration model. The condition of experiment simulation is shown in table 2.

\begin{tabular}{|c|c|c|}
\hline $\begin{array}{l}\text { Type of dynamic } \\
\text { model smoothing }\end{array}$ & Dynamic model & $\begin{array}{l}\text { Number of } \\
\text { parameters to be } \\
\text { estimated }\end{array}$ \\
\hline D1 & $\begin{array}{l}\text { With no experiential acceleration, } C_{D}, C_{R} \text { with } \\
\text { whole arc }\end{array}$ & 8 \\
\hline D2 & $\begin{array}{l}\text { With no experiential acceleration } \\
\qquad C_{D} / \text { 1hour }, C_{R} / 6 \text { hour }\end{array}$ & 20 \\
\hline D3 & $\begin{array}{l}\text { With traditional experiential acceleration } \\
\qquad C_{D} / \text { hour, } C_{R} / 6 \text { hour, } C_{R} / n^{*}\end{array}$ & 92 \\
\hline $\mathrm{D} 4$ & $\begin{array}{c}\text { With spline experiential } \\
\text { acceleration, } C_{D} / \text { 1hour, } C_{R} / 6 \text { hour, } C_{R} / 1 \text { hour }\end{array}$ & 136 \\
\hline
\end{tabular}

${ }^{*} \mathrm{n}$ is orbit period.

Table 2. Type of dynamic model smoothing.
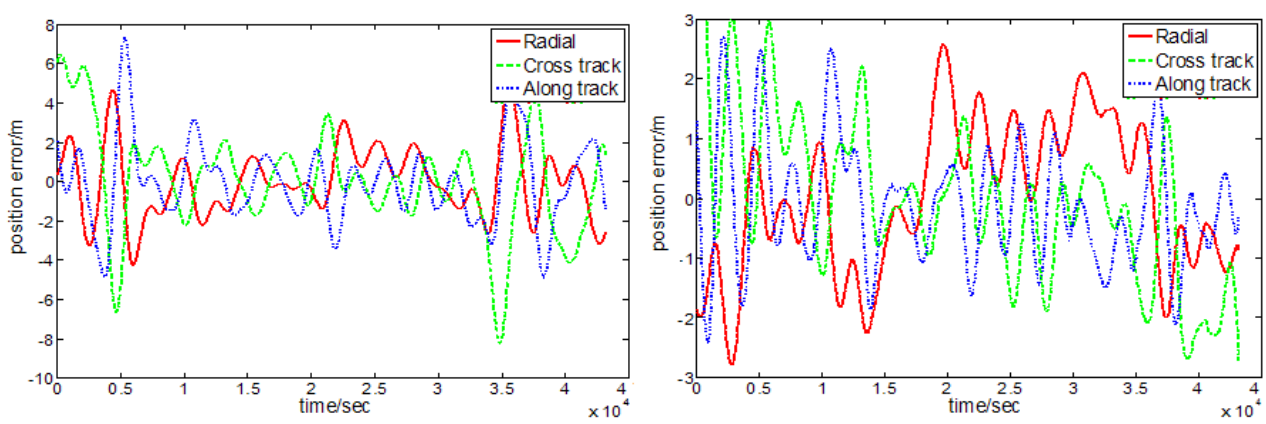

Fig. 8. Smoothing results of type D1 and D2.

From the above experiment, it can be drawn that the spline experiential acceleration model can effectively compensate the dynamic model error. In the type D1, the dynamic model with no experiential acceleration and with one group parameters cannot describe the actual orbit of CHAMP, so the smoothing error is high. In the type D2, although there is still no experiential model, the grouping parameters will absorb some error, the smoothing error is smaller than type D1. In the type D3, the smoothing error is smaller, and it is show that the traditional experiential acceleration model is effective. At the same time, the residual error means there are still some dynamic model error could not be explain by the traditional experiential acceleration, for the form of traditional experiential acceleration 1-cycle-per- 

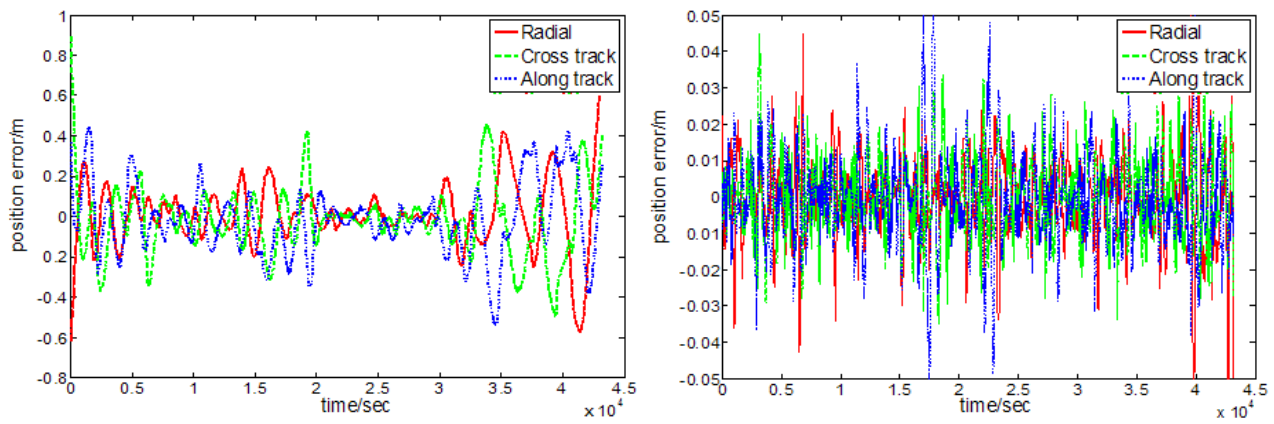

Fig. 9. Smoothing results of type D3 and D4.

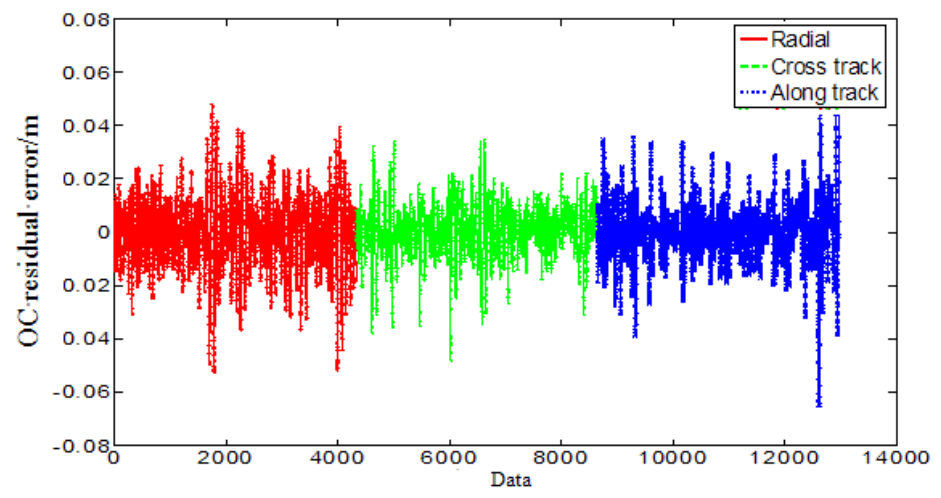

Fig. 10. The OC residual error of smoothing results of type D4.

revolution. At last, in the type D4, the spline model can explain dynamic error with any frequency, the error of smoothing is the most small in all the type experiments.

\section{References}

Tapley B D, Born G., Schutz B. (1986).Orbit Determination Fundamental And Application. Texas: The U niversity of Texas

Yunck T P, William G M , Thornton C L.(1985) .GPS Based Satellite Tracking System For Precise Positioning. IEEE Transactions on Geo science and Remote Sensing, Vol 23, pp 450 457.

Yunck T P, S C Wu, W I Bertiger, et al.(1994) .First Assessment of GPS-based Reduced Dynamic Orbiter Determination on Topex/Poseidon. Geophysics Research Letter (S0094-8276), Vol 21,pp 541-544.

Wu S C, T P Yunck, C L Thornton.(1991). Reduced-Dynamic technique for Precise Orbit Determination of Low Earth Satellites. Journal of Guidance, Control and Dynamics (S0371-5090), Vol 14,pp24-30. 
Xue Liugen, Han Jianguo.(2001). Asymptotic Properties of Two Stage Estimator in Semiparametric Regression Model. Appl Math J. Chinese Univ ser A, Vol16, pp 87-94.

Lin Lu, Cui Xia. (2006).Stahel -Donoho Kernel Estimator of model under nonparametric fixed design. Science in China ser.A. 2006,vol.36,pp 1156 1172. 


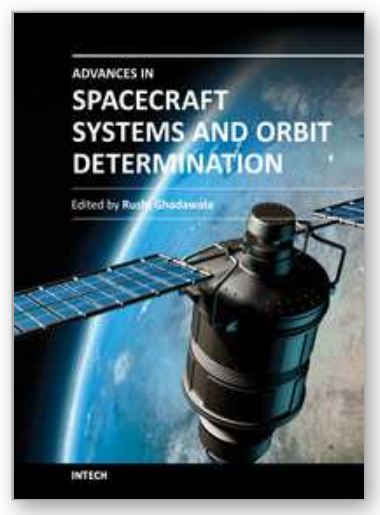

\author{
Advances in Spacecraft Systems and Orbit Determination \\ Edited by Dr. Rushi Ghadawala
}

ISBN 978-953-51-0380-6

Hard cover, 264 pages

Publisher InTech

Published online 23, March, 2012

Published in print edition March, 2012

"Advances in Spacecraft Systems and Orbit Determinations", discusses the development of new technologies and the limitations of the present technology, used for interplanetary missions. Various experts have contributed to develop the bridge between present limitations and technology growth to overcome the limitations. Key features of this book inform us about the orbit determination techniques based on a smooth research based on astrophysics. The book also provides a detailed overview on Spacecraft Systems including reliability of low-cost AOCS, sliding mode controlling and a new view on attitude controller design based on sliding mode, with thrusters. It also provides a technological roadmap for HVAC optimization. The book also gives an excellent overview of resolving the difficulties for interplanetary missions with the comparison of present technologies and new advancements. Overall, this will be very much interesting book to explore the roadmap of technological growth in spacecraft systems.

\title{
How to reference
}

In order to correctly reference this scholarly work, feel free to copy and paste the following:

Pan Xiaogang, Wang Jiongqi and Zhou Haiyin (2012). Research on the Method of Spacecraft Orbit Determination Based the Technology of Dynamic Model Compensation, Advances in Spacecraft Systems and Orbit Determination, Dr. Rushi Ghadawala (Ed.), ISBN: 978-953-51-0380-6, InTech, Available from:

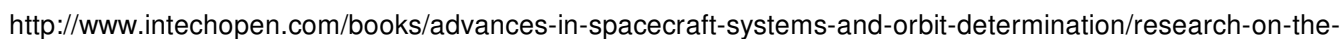
method-of-spacecraft-orbit-determination-based-the-technology-of-dynamic-model-compe

\section{INTECH}

open science | open minds

\section{InTech Europe}

University Campus STeP Ri

Slavka Krautzeka 83/A

51000 Rijeka, Croatia

Phone: +385 (51) 770447

Fax: +385 (51) 686166

www.intechopen.com

\section{InTech China}

Unit 405, Office Block, Hotel Equatorial Shanghai

No.65, Yan An Road (West), Shanghai, 200040, China

中国上海市延安西路65号上海国际贵都大饭店办公楼405单元

Phone: +86-21-62489820

Fax: $+86-21-62489821$ 
(C) 2012 The Author(s). Licensee IntechOpen. This is an open access article distributed under the terms of the Creative Commons Attribution 3.0 License, which permits unrestricted use, distribution, and reproduction in any medium, provided the original work is properly cited. 